\title{
Advances in Solid Core Photonic Bandgap Fiber Amplifiers
}

Alkeskjold, Thomas Tanggaard; Laurila, Marko; Petersen, Sidsel Rübner; Jørgensen, Mette Marie; Lægsgaard, Jesper; Olausson, Christina B.; Broeng, Jes

Published in:

OFC/NFOEC Technical Digest

Publication date:

2012

Document Version

Publisher's PDF, also known as Version of record

Link back to DTU Orbit

Citation $(A P A)$ :

Alkeskjold, T. T., Laurila, M., Petersen, S. R., Jørgensen, M. M., Lægsgaard, J., Olausson, C. B., \& Broeng, J. (2012). Advances in Solid Core Photonic Bandgap Fiber Amplifiers. In OFC/NFOEC Technical Digest (pp. OTh1H.1). Optical Society of America.

\section{General rights}

Copyright and moral rights for the publications made accessible in the public portal are retained by the authors and/or other copyright owners and it is a condition of accessing publications that users recognise and abide by the legal requirements associated with these rights.

- Users may download and print one copy of any publication from the public portal for the purpose of private study or research.

- You may not further distribute the material or use it for any profit-making activity or commercial gain

- You may freely distribute the URL identifying the publication in the public portal

If you believe that this document breaches copyright please contact us providing details, and we will remove access to the work immediately and investigate your claim. 


\title{
Advances in Solid Core Photonic Bandgap Fiber Amplifiers
}

\author{
Thomas T. Alkeskjold ${ }^{1}$, Marko Laurila ${ }^{2}$, Sidsel Petersen ${ }^{2}$, Mette Jørgensen ${ }^{1,2}$, Jesper Lægsgaard ${ }^{2}$, \\ Christina B. Olausson ${ }^{1}$, Jes Broeng ${ }^{1}$ \\ ${ }^{1}$ NKT Photonics, Blokken 84, DK-3460 Birkerød, Denmark \\ ${ }^{2}$ DTU Fotonik, Department of photonics engineering, Technical University of Denmark, Denmark \\ tta@nktphotonics.com
}

\begin{abstract}
We present recent development of photonic crystal fiber amplifiers containing photonic bandgap structures for enhanced spectral and modal filtering functionality.
\end{abstract}

OCIS codes: (060.0060) Fiber optics and optical communication; (060.3510) Lasers, Fiber

\section{Introduction}

The rapid development and deployment of high peak power and high pulse energy ytterbium-doped (Yb-doped) fiber amplifiers have been fuelled by the development of more and more complex Large Mode Area (LMA) fiber designs [1-6]. The demand for large effective area is driven by the need to mitigate nonlinear effects such as FourWave Mixing (FWM), Self-Phase Modulation (SPM), and Stimulated Raman Scattering (SRS), which can distort pulse amplification due to spectral and/or temporal broadening. To successfully apply fiber amplifiers, in for example semiconductor and solar cell scribing applications or frequency conversion applications, fiber amplifiers need to generate high beam quality with good pointing stability in order to deliver precise and stable performance. For this, Single-Mode (SM) fibers have a major advantage since Higher-Order Modes (HOMs) are suppressed by the fiber design and not by coiling or launch conditions. As fiber amplifiers are maturing other practical issues arise such as the need for compact fiber coils, beam delivery, suppression of Amplified Spontaneous Emission (ASE) and/or suppression of detrimental wavelengths generated through FWM, which can destroy the pumps since these wavelengths are not well isolated in standard pump reflectors.

Furthermore, fiber amplifiers operating with built-in spectral gain shaping has recently emerged as a novel method for generating non-standard wavelengths such as 589nm by frequency doubling an 1178nm Yb-doped amplifier [7, 8] or using cladding pumped Raman amplification. Both types of amplifiers require distributed spectral filtering to either suppress the ytterbium gain at lower wavelengths or to suppress the second Stokes wavelength to limit cascaded Raman processes [9].

Photonic BandGap (PBG) structures can be successfully incorporated into Photonic Crystal Fiber (PCF) amplifiers to provide suppression of Higher-Order Modes (HOMs), gain shaping, ASE and SRS suppression, as well as provide improved bending performance for achieving smaller form factors. In this paper, we present our latest results on LMA yb-doped fiber amplifiers having PBG structures enabling efficient amplification of high spectral brightness signals.

\section{Single-mode ROD PCF amplifiers}

Suppression of nonlinear effects is crucial for generating high peak power pulses in fiber amplifiers. The most common approach is to increase the effective mode area and increase the pump absorption, thereby reducing the effective nonlinear figure of merit. However, when increasing the core size of step-index fibers to beyond $15 \mu \mathrm{m}$ the core supports many spatial modes and this can significantly degrade the beam quality and cause beam instabilities. Fabricating large cores that only support one spatial mode requires tight index control to achieve the required low NA. Furthermore, tolerances on preform materials etc make production of low-NA fibers (approx <0.06) very challenging unless tolerances can be compensated during fiber draw.

Inset of figure 1a shows the endfacet of a so-called DMF rod fiber amplifier [6] having an $85 \mu \mathrm{m}$ core with $\sim 0.01 \mathrm{NA}$. The fiber core is formed by 19 index-matched yb-elements and the cladding is formed by 5 rings of airholes arranged in a hexagonal lattice. The cladding furthermore consists of 30 Germanium-doped rings arranged in kagome lattice within the hexagonal air lattice. These rings form precisely tuned Distributed Mode-Filtering (DMF) elements that filter HOMs out of the core and allows for SM operation. As with other resonant methods, these rings are tuned to filter HOMs in a specific wavelength region. Figure 1a shows a transmission plot of such as fiber. The oscillations in the spetrum are caused by mode beating between the Fundamental Mode (FM) and HOMs. Between 1050-1070nm there are no oscillations and the fiber core only supports one spatial mode. Below 1050nm, the FM couples to the DMF elements and becomes leaky (leaky regime).The pump absorption is $\sim 27 \mathrm{~dB} / \mathrm{m} @ 976 \mathrm{~nm}$, 
which gives an effective amplifier length of $70-90 \mathrm{~cm}$ depending on configuration. Figure $1 \mathrm{~b}$ shows the slope efficiency of a $70 \mathrm{~cm}$ rod amplifier in a wavelength-locked Q-switched laser configuration [10]. The rod delivers $14 \mathrm{~ns}$ pulses with up to $2 \mathrm{~mJ}$ pulse energy and $0.4 \mathrm{~nm}$ spectral width in a diffraction limited output with $\mathrm{M}^{2}<1.2$. The output beam has very high spectral brightness and was frequency doubled to $515 \mathrm{~nm}$ with $48 \%$ conversion efficiency.

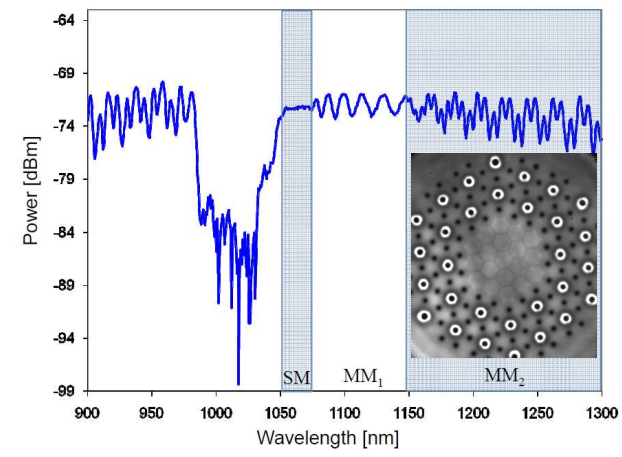

a)

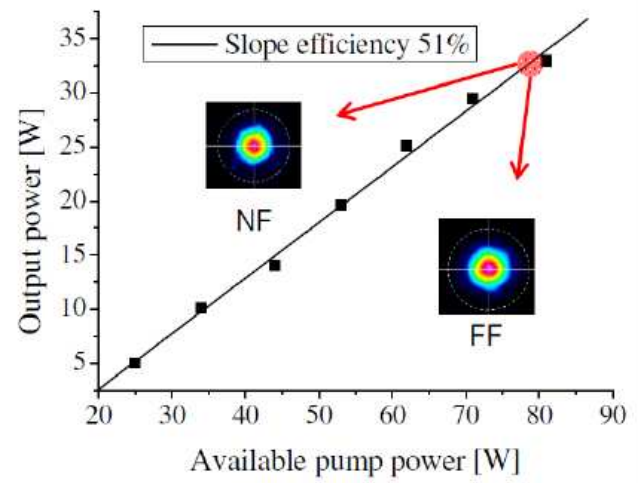

b)

Fig. 1. Transmission plot of the DMF rod fiber [6] (a). Inset shows a micrograph of the endfacet of the DMF rod fiber. Slope efficiency of the DMF rod fiber in a Q-switched laser cavity (b) [10].

Generating high-power ps pulses with high spectral brightness in fiber amplifiers is typically very challenging because SPM causes spectral broadening. However, recent results [11] have shown very efficient ps amplification using the DMF rod fiber in the leaky regime, delivering $>200 \mathrm{~W}$ of output with $>70 \%$ slope efficiency and $0.3 \mathrm{~nm}$ spectral width.

\section{Single-mode PBG PCF amplifiers}

Frequency doubling of lasers and amplifiers operating in the 1150-1200nm region produces yellow-orange light from 570-600nm. Yb-doped fibers have a very broad fluorescence spectrum extending up to $1200 \mathrm{~nm}$ and the smallsignal gain per unit length can be as high as $0.8 \mathrm{~dB} / \mathrm{m}$ hence the net gain can easily exceed $10 \mathrm{~dB}$ at $1178 \mathrm{~nm}$ [7]. However, the large gain between $1030 \mathrm{~nm}$ and $1100 \mathrm{~nm}$ creates very strong ASE and can lead to parasitic lasing. Parasitic lasing will limit the available gain at $1178 \mathrm{~nm}$ and thereby the power scalability. In order to overcome the problem of parasitic lasing, a full PBG structure provides wavelength-filtering and efficient suppression of ASE at the conventional ytterbium gain wavelengths between 1030 and $1100 \mathrm{~nm}$. The fiber structure is shown in the inset of figure 2a. The single-cell core consists of index-matched yb-doped silica and the cladding consists of Germaniumdoped high-index parabolic rods forming the PBG structure. Two boron rods on each side of the core create birefringence for maintaining the polarization through the fiber. As shown in figure 2a, using 40meters of fiber, sufficient gain is accumulated and $167 \mathrm{~W}$ of output power has been generated at $1178 \mathrm{~nm}$ with a slope efficiency of $61 \%$ [8]. Figure 2b shows the transmission spectrum of the PBG fiber together with the amplified output spectrum. As shown, the fiber efficiently suppresses gain below $1178 \mathrm{~nm}$ and allows for $16 \mathrm{~dB}$ of net gain at $1178 \mathrm{~nm}$ [8].

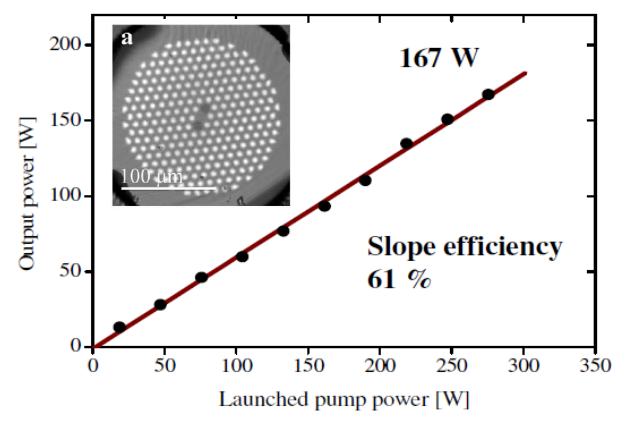

a)

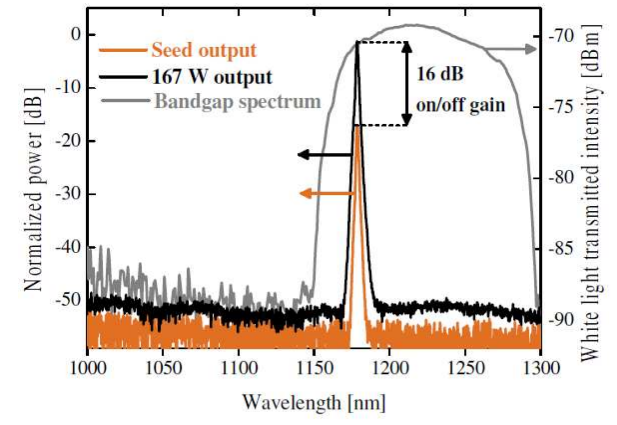

b)

Fig. 2. Slope efficiency for $1178 \mathrm{~nm}$ amplification, inset shows micrograph of the endfacet of the PBG fiber [8] (a). Transmission plot of the PBG fiber and the amplified output spectrum [8] (b). 


\section{Single-mode hybrid PCF amplifiers}

Full PBG structures provide excellent gain filtering, but the inherent all-solid structure makes core size scaling difficult if SM performance is to be maintained. To scale the core size, and thereby the effective mode area, hybrid PCF structures are more promising [12]. The cladding of hybrid PCF structures contains several rings of airholes as conventional PCFs but some of the airholes have been replaced with Germanium-doped high-index PBG elements to provide wavelength filtering. Two different hybrid PCF structures are shown in figure 3 . The fibers in figure 3 contain two groups of Germanium-doped high-index rods arranged in either a single row or with three adjacent rows. The designs are asymmetric, which means that the diameter of the rods on the left and right side of the core are not identical. In these cases, the right row of rods is scaled to $\sim 85 \%$ of the left row of rods. This asymmetry provides one more degree of freedom in the design of the wavelength filtering, and it is possible to further narrow the PBG spectrum down to $\sim 3-4 \%$ spectral width and provide very robust bending performance with $10-15 \mathrm{~cm}$ bending diameter possible for core sizes of 35-45 $\mu \mathrm{m}$ [13], while maintaining SM performance.

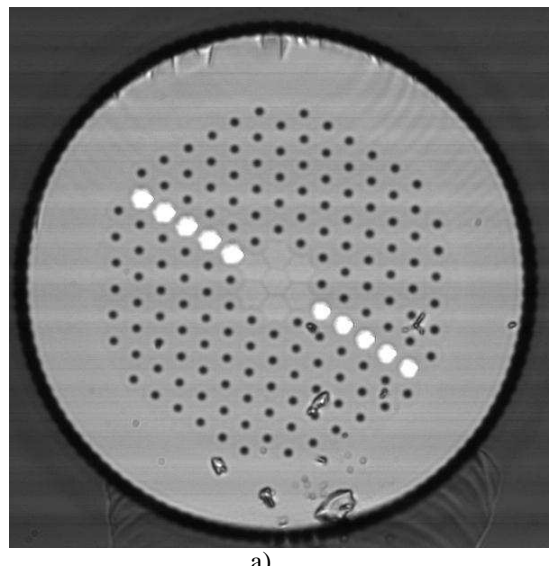

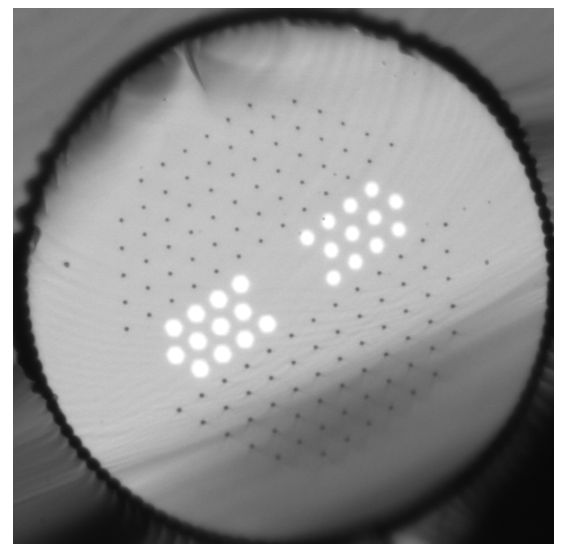

b)

Fig.3. Micrographs of hybrid PCF fibers having one-row (a) and three-rows (b) of PBG elements for enhanced spectral filtering.

\section{References}

[1] J. Limpert et al, "Extended single-mode photonic crystal fiber lasers," Opt. Express 14, 2715-2720 (2006).

[2] C.D. Brooks and F. Di Teodoro, "Multi-megawatt peak-power, single-transverse-mode operation of a $100 \mu \mathrm{m}$ core diameter, Yb-doped rodlike photonic crystal fiber amplifier” Appl. Phys. Lett. 89(11), 111119-111121 (2006).

[3] C-H. Liu et al, "Chirally Coupled Core Fibers at 1550-nm and 1064-nm for Effectively Single-Mode Core Size Scaling", paper CTuBB3 at CLEO/QELS 2007, Baltimore.

[4] L. Dong et al, "Ytterbium-doped all glass leakage channel fibers with highly fluorine-doped silica pump cladding," Opt. Express 17, 89628969 (2009).

[5] F. Jansen et al, "The influence of index-depressions in core-pumped Yb-doped large pitch fibers," Opt. Express 18, 26834-26842 (2010).

[6] T. T. Alkeskjold et al, "Single-mode ytterbium-doped large-mode-area photonic bandgap rod fiber amplifier," Opt. Express 19, 7398-7409 (2011).

[7] A. Shirakawa et al, "High-power Yb-doped photonic bandgap fiber amplifier at 1150-1200 nm," Opt. Express 17, 447-454 (2009).

[8] C. B. Olausson et al, "167 W, power scalable ytterbium-doped photonic bandgap fiber amplifier at 1178nm," Opt. Express 18, 16345-16352 (2010).

[9] B. Ward, "Solid-core photonic bandgap fibers for cladding-pumped Raman amplification," Opt. Express 19, 11852-11866 (2011).

[10] M. Laurila et al, "Q-switching and efficient harmonic generation from a single-mode LMA photonic bandgap rod fiber laser," Opt. Express 19, 10824-10833 (2011).

[11] M. Laurila et al, " Efficient high power fiber amplifier utilizing the single mode distributed mode filtering bandgap rod fiber" Proceedings of SPIE Photonics West 2012, to be published.

[12] T. T. Alkeskjold, "Large-mode-area ytterbium-doped fiber amplifier with distributed narrow spectral filtering and reduced bend sensitivity," Opt. Express 17, 16394-16405 (2009).

[13] S.Petersen et al, "Ytterbium-doped large-mode-area photonic crystal fiber amplifier with gain shaping for use at long wavelengths" Proceedings of SPIE Photonics West 2012, to be published. 